\title{
Revised time-of-flight calculations for high-latitude geomagnetic pulsations using a realistic magnetospheric magnetic field model
}

\author{
J. A. Wild ${ }^{1}$ and T. K. Yeoman \\ Department of Physics and Astronomy, University of Leicester, Leicester, UK \\ C. L. Waters \\ School of Mathematical and Physical Sciences, University of Newcastle, Callaghan, New South Wales, Australia \\ Received 7 December 2004; revised 9 May 2005; accepted 18 August 2005; published 11 November 2005.
}

[1] We present a simple time-of-flight analysis of Alfvén pulsations standing on closed terrestrial magnetic field lines. The technique employed in this study in order to calculate the characteristic period of such oscillations builds upon earlier time-of-flight estimates via the implementation of a more recent magnetospheric magnetic field model. In this case the model employed is the Tsyganenko (1996) field model, which includes realistic magnetospheric currents and the consequences of the partial penetration of the interplanetary magnetic field into the dayside magnetopause. By employing a simple description of magnetospheric plasma density, we are therefore able to estimate the period of standing Alfvén waves on geomagnetic field lines over a significantly wider range of latitudes and magnetic local times than in previous studies. Furthermore, we investigate the influence of changing season and upstream interplanetary conditions upon the period of such pulsations. Finally, the eigenfrequencies of magnetic field lines computed by the time-of-flight technique are compared with corresponding numerical solutions to the wave equation and experimentally observed pulsations on geomagnetic field lines.

Citation: Wild, J. A., T. K. Yeoman, and C. L. Waters (2005), Revised time-of-flight calculations for high-latitude geomagnetic pulsations using a realistic magnetospheric magnetic field model, J. Geophys. Res., 110, A11206, doi:10.1029/2004JA010964.

\section{Introduction}

[2] Since Dungey [1954] proposed for the first time that the long-period geomagnetic pulsations observed on the ground might be the result of standing Alfvén waves being excited on geomagnetic field lines, a wealth of groundbased and satellite observations have established the importance of field line oscillations, and it has become recognized that such waves transfer both energy and momentum through the coupled magnetosphere-ionosphere system. These processes are most significant in the high-latitude ionosphere, where the magnetosphere-ionosphere interaction is strongest. The waves also act as an important diagnostic of magnetospheric morphology and dynamics.

[3] In general, ultra-low frequency (ULF) waves have an energy source external to the Earth, such as an impulse in the solar wind, solar wind buffeting, or the Kelvin-Helmholtz instability on the magnetopause. However, the sequence of events which connect such broadband boundary oscillations to the resonant driving of an individual field line

\footnotetext{
${ }^{1}$ Now at Department of Communication Systems, Lancaster University, Lancaster, UK.

Copyright 2005 by the American Geophysical Union. 0148-0227/05/2004JA010964\$09.00
}

at its natural eigenfrequency is not straightforward. Initially, a direct coupling between the wave source on the boundary and a local field line was proposed, which successfully explained many observed features of field line resonances [e.g., Southwood, 1974]. The observed discrete frequency spectrum remained a puzzle, however, and subsequently global compressional oscillations of the magnetosphere, setting the cavity formed by magnetospheric boundaries into oscillation, were suggested as a cause of a local field line resonating at its characteristic frequency by Kivelson et al. [1984]. In this scenario the field line driven to resonance was one whose eigenfrequency matched a natural frequency of the magnetospheric cavity. More recently, it has become accepted that the magnetospheric cavity oscillation is better described as a magnetospheric waveguide, with the characteristic frequencies of the waveguide coupling to field line oscillations [e.g., Harrold and Samson, 1992; Samson et al., 1992; Rickard and Wright, 1994]. Such a waveguide mode may be excited directly by buffeting of the magnetosphere or through a broadband wave source on the magnetopause [e.g., Mann et al., 1999]. However, experimental evidence for plasmatrough cavity/waveguide modes remains sparse [e.g., Waters et al., 2002].

[4] In understanding observations of field line oscillations, a knowledge of the expected eigenperiods of the field 
lines as a function of magnetic latitude, local time, and season is clearly of vital importance. At middle and low latitudes, where the magnetic field is close to dipolar, theoretical estimates of field line eigenperiods have been computed by Cummings et al. [1969] and Sinha and Rajaram [1997], for both the toroidal and polodial modes of oscillation. In such calculations a hydrogen plasma is assumed. At low and equatorial latitudes, where heavier species such as oxygen and helium take over, similar calculations have been presented by Poulter et al. [1988]. At high latitudes, deviations from a dipolar field become significant. Such complexities in the field geometry prevent an analytical treatment of the eigenperiods, and this problem was addressed via a simple time-of-flight approach by Warner and Orr [1979]. Warner and Orr [1979] used the Mead and Fairfield [1975] model of the terrestrial magnetic field, which allowed calculations of field line eigenperiods up to latitudes of $\sim 75^{\circ}\left(\sim 68^{\circ}\right)$ in the noon (midnight) sector and also allowed the effects of magnetic activity and dipole tilt to be investigated. The Warner and Orr [1979] time-offlight approach to eigenmode calculations at high latitude has been used extensively in the interpretation of ULF wave observations. Their results were invoked in the interpretation of early results from auroral zone and subauroral VHF coherent radar measurements of ULF waves [e.g., Villain, 1982; Yeoman and Lester, 1990]. The results have also provided a baseline for the explanation of the local time variations of magnetic field oscillations at Pc5 frequencies observed in ground-based data [Yumoto and Saito, 1983; Glassmeier and Stellmacher, 2000]. The technique has been equally important in the interpretation of the observed periods in ground-based data at very high magnetic latitudes near the cusp region [Ables et al., 1988; Waters et al., 1995; Clauer et al., 1997] and similarly in high-latitude spacecraft observations out to $L$ shells up to $L=9$ [e.g., Anderson et al., 1989, 1990]. In addition to these experimental studies, the modeling of Warner and Orr [1979] has also been used to provide baseline eigenfrequencies for ULF wave modeling efforts in realistic geometries [e.g., Allan et al., 1986; Lee and Lysak, 1990; Wright, 1992].

[5] The eigenfrequencies of ULF waves provide an important diagnostic of the plasma loading along the field line, both within the plasmasphere and the plasmatrough [e.g., Poulter et al., 1984; Waters et al., 1996; Loto'aniu et al., 1999; Dent et al., 2003]. At higher latitudes the interpretation of such data in terms of plasma loading clearly requires a realistic model of the magnetic field geometry as a prerequisite for any accurate inference of the plasma density.

[6] Since the original calculations of Warner and Orr [1979], great advances have been made in magnetic field modeling, and the limitations of the Mead and Fairfield [1975] model have become clear. The location of the open/ closed field line boundary and magnetic field stretching in the midnight sector are two particularly clear instances where the Mead and Fairfield [1975] model requires updating. Recently, Rankin et al. [2000] solved the fundamental eigenmode equations under the magnetic field geometry of the Tsyganenko [1996] magnetic field model [Tsyganenko, 1995, 1996] in the midnight sector, demonstrating that frequencies could result which were an order of magnitude lower than those expected for a dipole field.
Here we present a refinement of the simpler time-of-flight approximation of Warner and Orr [1979], based upon the Tsyganenko [1996] magnetic field model in order to explore such effects under a wide variety of latitude, local time, dipole tilt, and magnetic activity conditions.

\section{Models}

[7] Approximating the oscillations on terrestrial field lines as Alfvén waves standing on individual field lines, the period of oscillation, $\tau$, can be approximated by the time-of-flight approximation

$$
\tau=2 \int \frac{\mathrm{ds}}{V_{A}}
$$

where $V_{A}$ is the Alfvén velocity, and the integration is carried out over the entire length of the field line. The Alfvén speed at some position on the field lines is described by

$$
V_{A}^{2}=\frac{B^{2}}{\mu_{0} \rho}
$$

where $B$ and $\rho$ denote the local magnetic field strength and plasma density, respectively. Therefore in order to calculate the period of an Alfvénic oscillation on a terrestrial field line, it is necessary to employ realistic descriptions of the magnetospheric magnetic field and plasma environment.

\subsection{Magnetic Field Model}

[8] For the purposes of this investigation, we have implemented the Tsyganenko [1996] magnetic field model [Tsyganenko, 1995, 1996], hereafter referred to as the "T96" model. This model has several significant advantages over the magnetospheric field model of Mead and Fairfield [1975] employed by Warner and Orr [1979]. Most importantly for the current study, the T96 model describes the Earth's magnetotail much more realistically than that of Mead and Fairfield [1975] which extended to only $17 R_{E}$ downstream of the planet. Furthermore, the magnetic field described by the T96 model is derived from several modular elements representing magnetic field sources within the magnetopause current system, the ring current, the crosstail current sheet, and Region 1 and 2 Birkeland current systems, in addition to the Earth's internal field. As such the T96 model contains none of the built-in dawn-dusk and north-south symmetries found within the earlier model and allows for a more realistic description of local time effects. An additional key difference between the field models of Tsyganenko [1995, 1996] and Mead and Fairfield [1975] is that the former is parameterized by the Dst index, the solar wind dynamic pressure, and the $B_{Y}$ and $B_{Z}$ components of the IMF incident upon the magnetopause, whereas the latter relies only upon a crude parameterization by the $K_{P}$ index.

\subsection{Plasma Model}

[9] In order to investigate the implementation of a more realistic magnetospheric magnetic field model upon the results of Warner and Orr [1979], we have retained the plasma density model utilized in that study. This model, based upon data reviewed by Chappell [1972], is summarized in Table 1. The in situ magnetospheric plasma densi- 
Table 1. Plasma Density Models for Various Magnetospheric Regions (Adapted From Warner and Orr [1979]) ${ }^{\mathrm{a}}$

\begin{tabular}{lcc}
\hline \multicolumn{1}{c}{ Region } & $\begin{array}{c}\text { Typical Density } \\
\text { at } L=4, \text { ions } \mathrm{cm}^{-3}\end{array}$ & $\begin{array}{c}\text { Equatorial } \\
\text { Density Variations }\end{array}$ \\
\hline Extended plasmasphere $_{\text {busk plasmasphere }}$ & 450 & $R^{-3}$ \\
Dlasmatrough $^{\mathrm{c}}$ & 188 & $R^{-4}$ \\
0800 MLT & 15 & $R^{-4}$ \\
1400 MLT & 60 & $R^{-3}$ \\
Detached plasma & & $R^{-4}$ \\
Upper limit & 400 & $R^{-4}$ \\
Lower limit & 100 & \\
\hline
\end{tabular}

${ }^{\mathrm{a}}$ Minimum density $=0.01$ ions $\mathrm{cm}^{-3}$.

${ }^{\mathrm{b}}$ Limited to $1500-2100$ MLT.

${ }^{c}$ MLT dependence described by Chappell et al. [1971]; see text for details.

ties are drawn from OGO 5 mass spectrometer measurements during 1968-1969. The distribution of magnetospheric plasma along individual field lines at distances greater than $2 R_{E}$ can then be represented as proportional to $r^{-n}$, with the exact value of $n$ depending upon the chosen region of the magnetosphere We have departed from this is in only two regards. First, the description of the plasma density within the plasmatrough given by Warner and Orr [1979], which is local time dependant due to filling from the ionosphere during the day, is insufficiently detailed to allow full implementation in this model. We have therefore reverted to the plasmatrough plasma density distribution predicted by Chappell et al. [1971] assuming a constant upward flux of $3 \times 10^{8}$ ions $\mathrm{cm}^{-2} \mathrm{~s}^{-1}$ with a collisionless distribution of ionization along the field line. Second, due to the extended tail of the T96 model, compared to that of Mead and Fairfield [1975], it has been necessary to introduce a minimum magnetospheric plasma density in order to avoid excessively large Alfvén speeds in the distant tail. For this study, we have therefore set the minimum plasma number density to be 0.01 ions $\mathrm{cm}^{-3}$.

\section{Results}

[10] Figure 1 presents an overview of the estimated variation in Alfvén pulsation period as a function of geomagnetic latitude under a variety of conditions. In order to gauge the accuracy of the time-of-flight method employed in this study, we compare the results with those from a numerical solution to the wave equations approach under identical geomagnetic conditions. Each panel of Figure 1 presents a pulsation period-latitude profile calculated using the time-of-flight approximation applied to the T96 model (solid line), the same approach applied to a purely dipolar field (dotted line), and a numerical solution

Figure 1. The variation of pulsation period with geomagnetic latitude, calculated using the time-of-flight approximation (solid lines) and the Singer et al. [1981] numerical technique (dashed lines) based upon the T96 magnetospheric model. In each case the model is parameterized as indicated. An equivalent period-latitude profile, computed using a time-of-flight analysis based upon a simple dipolar field and an identical magnetospheric plasma distribution, is shown for comparison (dotted line). (a)

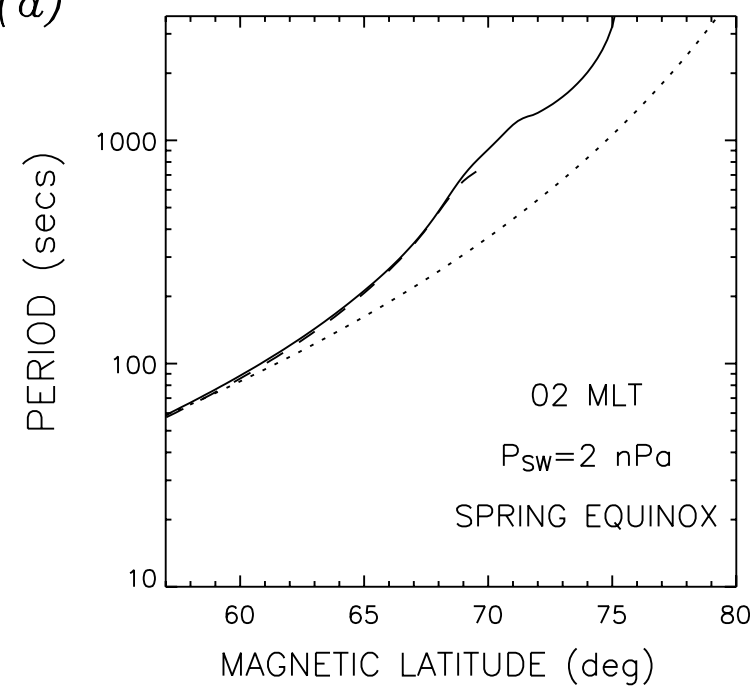

(b)

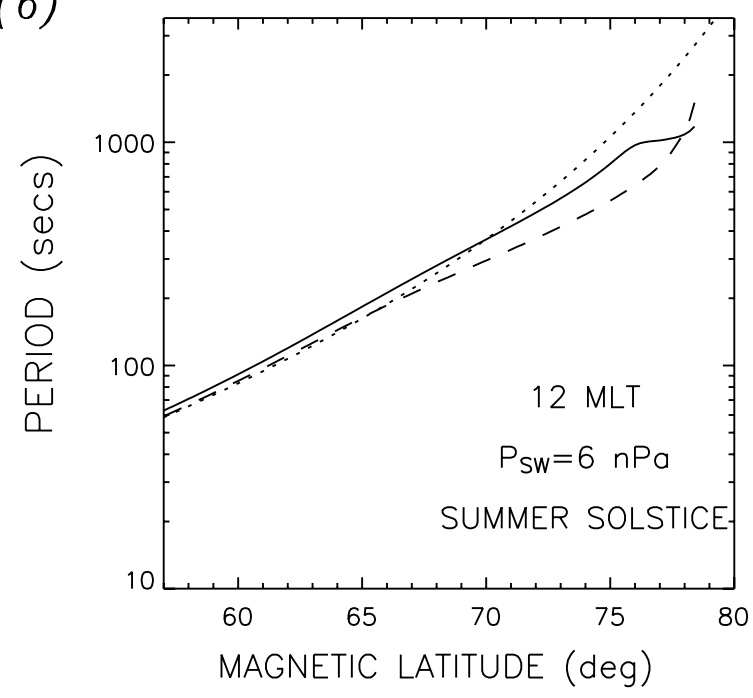

(c)

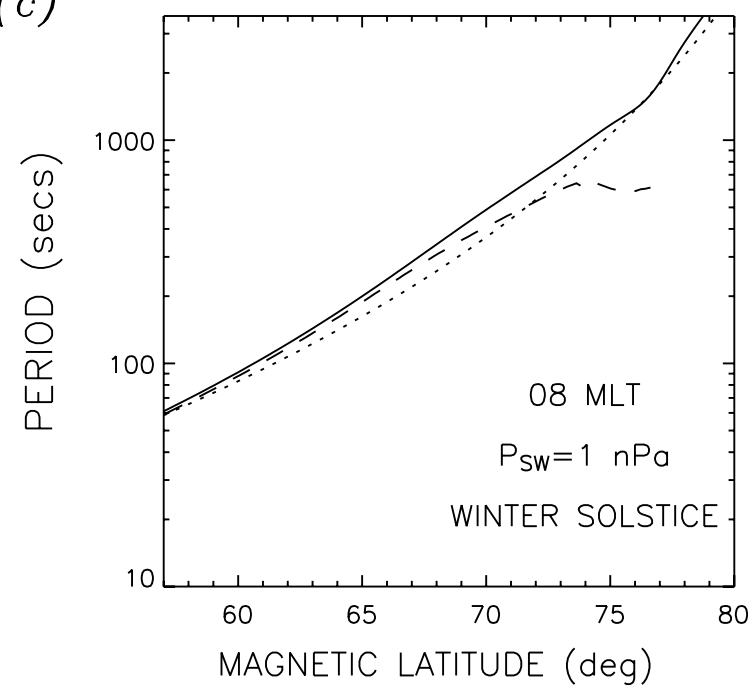


of the wave equations based upon the application of the equations of Singer et al. [1981] to the T96 model (dashed line). The wave equation described by Singer et al. [1981] was numerically solved using a fourth-order Runge-Kutta algorithm as described by Waters et al. [1995]. In order to numerically solve the Singer equation, it is necessary to compute the geometry of adjacent field lines to obtain the $h_{\alpha}$ parameter. At high latitudes, the field tracing for one field line may track to the opposite hemisphere, while the other tracks an open field line. Furthermore, the separation between adjacent field lines can become large along some sections with questionable results from the numerical integration. Any results that exhibited field tracing difficulties were rejected.

[11] In each panel of Figure 1, the plasma model employed is identical in order to emphasize the variations due to differing magnetic field configurations. More specifically, we have defined the plasma density at $L=4$ to be 100 ions $\mathrm{cm}^{-3}$, decreasing with radial distance in the equatorial plane with a $r^{-4}$ dependence. In all panels, three of the four T96 input parameters remain constant (Dst $=$ $0 \mathrm{nT}$, IMF $B_{Y}=\mathrm{IMF} B_{Z}=0 \mathrm{nT}$ ).

[12] Figure 1a corresponds to the pulsation period in the 0200 MLT magnetic meridian under spring equinox conditions (equivalent to $0^{\circ}$ dipole tilt) with the solar wind dynamic pressure $\left(P_{S W}\right)$ set at $2 \mathrm{nPa}$. In this configuration, the time-of-flight (solid line) and Singer et al. [1981] (dashed line) estimates of field line eigenfrequency are in almost exact agreement in the region where both are calculated. Furthermore, both approaches based upon the T96 model estimate the eigenperiod to be significantly (in excess of $300 \%$ ) larger than the time-of-flight estimate for a dipolar field geometry (dotted line). This is as expected given that the dipolar magnetic field is a wholly unrealistic description of the midlatitude/high-latitude magnetic field geometry in the magnetotail.

[13] Figure 1b corresponds to the 1200 MLT (noon) meridian at northern hemisphere summer solstice (equivalent to $\sim 34^{\circ}$ dipole tilt) with $P_{S W}=6 \mathrm{nPa}$, while Figure 1c corresponds to the 0800 MLT magnetic meridian under winter solstice conditions (equivalent to $\sim-34^{\circ}$ dipole tilt) with $P_{S W}=1 \mathrm{nPa}$. In both cases, the agreement between the two approaches is generally excellent at magnetic latitudes equatorward of $\sim 70^{\circ}$ Mlat (the numerically calculated figure being $\sim 75 \%$ of the time-of-flight equivalent). At latitudes poleward of this, the difference between the values increases (the equivalent comparison being $\sim 65 \%$ in Figure $1 \mathrm{~b}$ at $75^{\circ} \sim$ Mlat). However, we note that in the case of Figure $1 \mathrm{~b}$, the estimates then converge at $\sim 78^{\circ}$. Therefore the time-of-flight approach (when applied to the T96 model field) yields estimates that are broadly in agreement with the Singer et al. [1981] numerical technique. While at very high latitudes (poleward of $\sim 75^{\circ}$ Mlat) the difference between the two estimates may be significant ( $\sim$ several tens of percent), it is difficult to determine which technique is the most accurate. Given that the time-of-flight approach allows the rapid investigation of Alfvén pulsations over a larger range of latitudes than the Singer et al. [1981] technique (subject of course to the accuracy of the magnetic field model employed), we shall use it to investigate the latitudinal, diiurnal, seasonal variations in the frequency/ period of Alfvénic pulsations on geomagnetic field lines. In the final section our estimates will be compared to field line oscillations presented in investigations by other authors.

\subsection{Variation of Period With Latitude}

[14] Figures 2a, 2b, and 2c present the latitudinal variations in Alfvén pulsation period under equinox conditions between $57^{\circ}-80^{\circ}$ magnetic latitude along the 0800 MLT, 1600 MLT, and 0000 MLT magnetic meridians, respectively. In all cases the T96 input parameters were set to $P_{S W}=$ $2 \mathrm{nPa}, D s t=0 \mathrm{nT}$, IMF $B_{Y}$ and IMF $B_{Z}=0 \mathrm{nT}$ such that the configuration of the magnetospheric field remained constant. In each panel the results of time-of-flight analysis for the plasma regions listed in Table 1 are presented.

[15] As expected, the characteristic period of Alfvénic micropulsations generally increases with magnetic latitude in all local time sectors, irrespective of the plasma distribution selected. Figure $2 \mathrm{a}$ is broadly consistent with previous results under similar geomagnetic conditions. However, our calculations improve upon those of Warner and Orr [1979] in several regards. Most significantly, the more realistic magnetic field model employed allows us to extend our time-of-flight calculations to magnetic field lines that originate at higher latitudes. For example, we note that in this local time sector, the calculations of Warner and Orr [1979] were limited to magnetic latitudes $<73^{\circ}$. Comparison of Figures $2 \mathrm{a}$ and $2 \mathrm{~b}$ reveals slight differences between the postdawn and predusk sectors (in addition to the extra dusk plasmasphere trace), the consequence of local time asymmetries contained within the T96 magnetic field model but omitted from the Mead and Fairfield [1975] model employed by Warner and Orr [1979]. Furthermore, meridional latitude-period profiles originating from the southern hemisphere (not shown) indicate small north-south asymmetries, also not included in the Mead and Fairfield [1975] model.

[16] The final latitude-period profile presented here corresponds to the midnight meridian (Figure 2c). These profiles represents a significant departure from the estimated pulsation period-latitude relationships proposed by Warner and Orr [1979] due to the extended nature of the magnetotail described by the T96 model. While the Mead and Fairfield [1975] model was valid only within $17 R_{E}$ of the Earth, the equatorial crossing points of field lines in the midnight sector are predicted to be located much further down tail at latitudes poleward of $\sim 68^{\circ}$ Mlat. For the purposes of this investigation, we have designated any magnetic field lines that extend further than $300 R_{E}$ from the Earth as "open." The local maximum in each latitudeperiod profile (located at $\sim 68^{\circ}$ Mlat) is the result of the rapidly increasing field line length at increasingly northward positions on the midnight meridian. This effect, a consequence of T96 model configuration, is indicated in Figure 2c: the variation of field line length in the midnight sector, plotted as a function of magnetic latitude, is indicated by the dashed line, according to the scale on the right-hand axis. This effect is limited in azimuth to approximately \pm 2 hours MLT either side of midnight.

\subsection{Variation of Period With MLT}

[17] Figure 3 indicates the variations in micropuslation period for the different plasma regions at equinox as a function of MLT at $68^{\circ}$ (Figure $3 \mathrm{a}$ ), $72^{\circ}$ (Figure $3 \mathrm{~b}$ ), and $76^{\circ}$ 

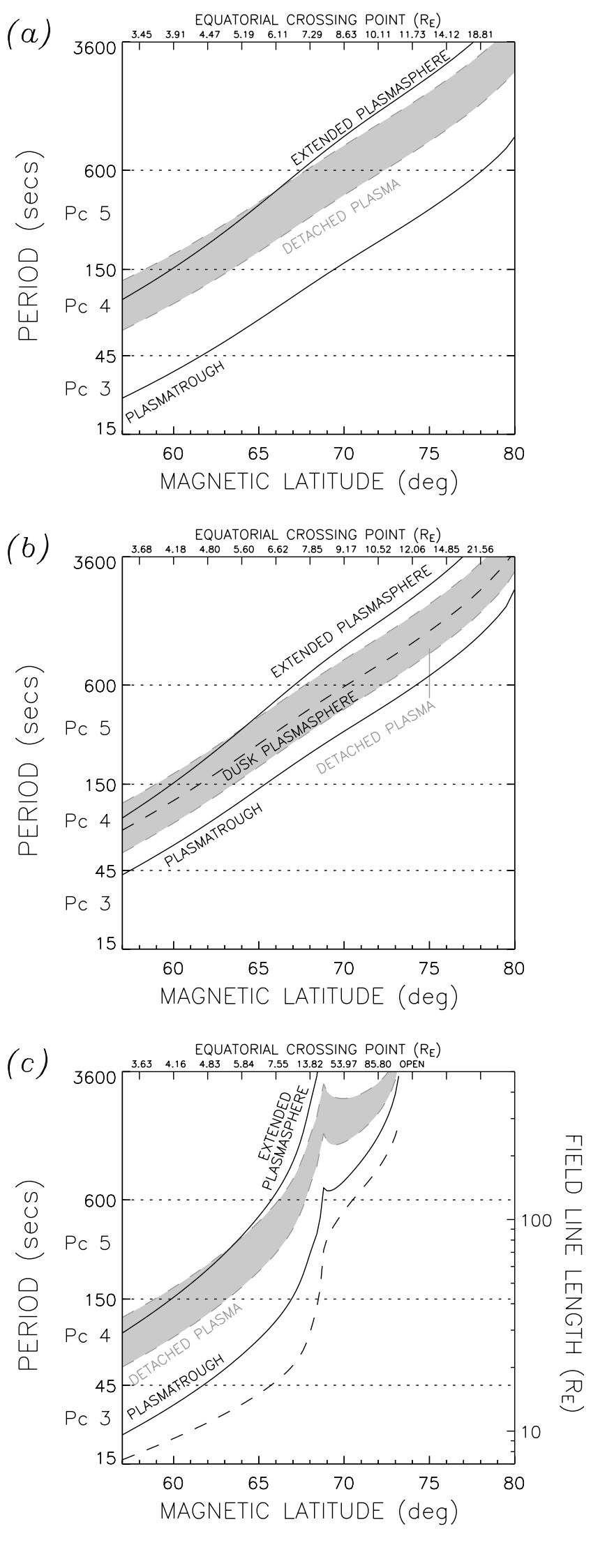

Figure 3. The variation of pulsation period as a function of magnetic local time under equinox conditions for terrestrial field lines at (a) $68^{\circ}$, (b) $72^{\circ}$, and (c) $76^{\circ}$ Mlat.

(Figure 3c) Mlat. As in previous cases, the T96 input parameters were set to $P_{S W}=2 \mathrm{nPa}, D s t=0 \mathrm{nT}$, IMF $B_{Y}=$ IMF $B_{Z}=0 \mathrm{nT}$. The results shown in Figure $3 \mathrm{a}$ are consistent with those presented by Warner and Orr [1979]. In the extended plasmasphere, dusk plasmasphere,

Figure 2. Magnetic latitude-period profiles for various plasma distributions at (a) 0800 MLT, (b) 1600 MLT, and (c) 0000 MLT under equinox conditions. The radial displacement of the equatorial crossing point of magnetic field lines are indicated in each case. In Figure $2 \mathrm{c}$ the variation of magnetic field line length in the midnight sector described by the T96 model is also plotted as a function of magnetic latitude under equinox conditions (dashed line). 


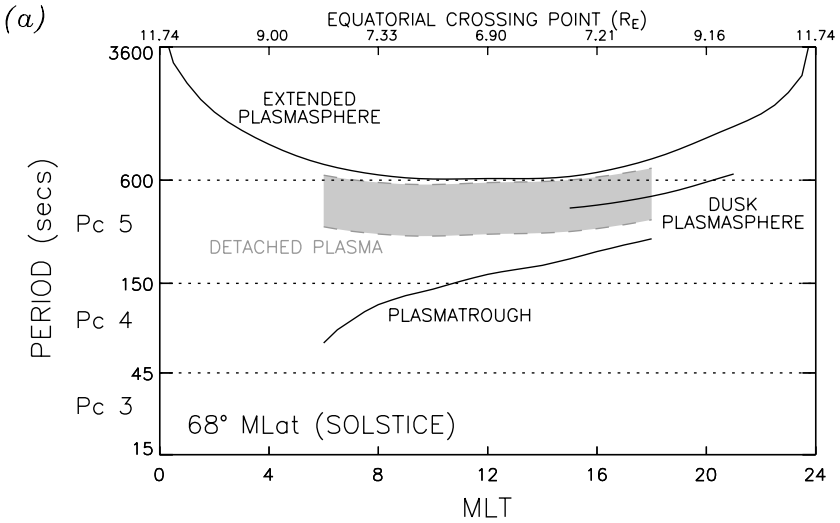

(b)
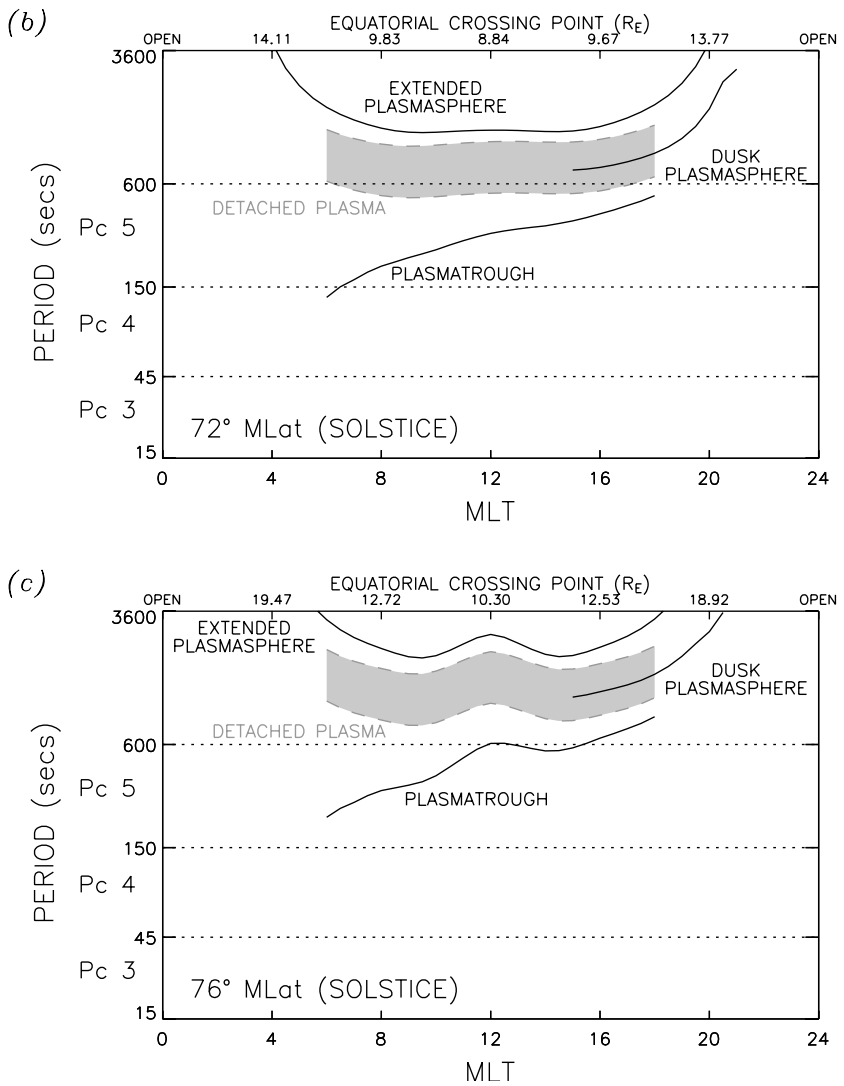

Figure 4. The variation of pulsation period as a function of magnetic local time under northern hemisphere summer solstice conditions for terrestrial field lines at (a) $68^{\circ}$, (b) $72^{\circ}$, and (c) $76^{\circ}$ Mlat.

and detached plasma regions, no local time dependencies are included in the model plasma distributions. As such, the profiles are influenced mainly by the changing shape of the field lines at this latitude. Each therefore exhibits a characteristic u-shaped profile, with the minimum period being located at noon, where the field lines are shortest and the field strength strongest. For clarity, the profiles corresponding to the dusk plasmasphere, detached plasma, and plasmatrough models are only plotted over a physically reasonable range of local times. We note that between noon and midnight local times, the equatorial crossing point of the $68^{\circ}$ Mlat field line varies by $\sim 6 R_{E}$. This contrasts with the $\sim 2 R_{E}$ variation predicted by the Mead and Fairfield [1975] magnetic field model. Therefore the correspondence between these results and those of Warner and Orr [1979], although excellent at all local times, is closest in the noon sector, where the magnetic field models used in each case are most alike.

[18] Unlike the other plasma regions, the density distribution model employed for the plasmatrough is inherently dependent upon local time. The estimated period-MLT profile for this region is therefore markedly different. In this case the plasma density variations at different local times are more significant than the change in the length and shape of the $68^{\circ}$ Mlat field line. The pulsation period is lowest in the predawn sector (when the plasma in the trough is relatively rarefied) and highest in the postdusk sector (when the plasma density within the trough has been enhanced due to filling from the dayside ionosphere).

[19] Figures $3 \mathrm{~b}$ and $3 \mathrm{c}$ present corresponding periodMLT profiles at $72^{\circ}$ and $76^{\circ}$ Mlat, respectively. In each case the period-MLT curves have been systematically shifted upward due to the increasing lengths of field lines at higher latitudes. As indicated by the equatorial crossing points of field lines at these higher latitudes, Alfvénic micropulsations travel significantly greater radial distances from the Earth than considered by Warner and Orr [1979]. Note also the slight local maximum in pulsation period in the postnoon sector $(\sim 1400$ MLT) at the highest latitude (Figure 3c).

\subsection{Variation of Period With Season/Dipole Tilt}

[20] Figure 4 presents identical period-MLT profiles as those shown in Figure 3, except the calculations have been made under northern hemisphere summer solstice conditions rather than an equinox configuration (i.e., $\sim 34^{\circ}$ dipole tilt angle rather than $0^{\circ}$ ). The T96 model solar wind and IMF parameterization remains unchanged. It is immediately apparent that the overall trends are generally unaffected by this change, although detailed examination reveals some small differences. Essentially, at solstice the pulsation period is reduced slightly in the daytime and increased during the nighttime. This is a consequence of the slight compression of the dayside magnetosphere as the pole tilts toward the Sun. The reduced field line length and enhanced field strength combined to increase the propagation speed of Alfvén pulsation, thus reducing the pulsation period. In the tail the opposite effect occurs the field lines being drawn further out downstream in the T96 model. The increase in tail field line length and reduction of the tail field strength (which reduces the Alfvén speed) results in increased pulsation periods. As was the case at equinox (Figure 3c), we note that at the highest latitude presented $\left(76^{\circ}\right.$ Mlat), there is local maximum in the predicted oscillation period, although at solstice it is much more pronounced and centered at noon.

\subsection{Variation of Period With Solar Wind and IMF Conditions}

[21] Finally, we present the impact of different solar wind and IMF parameterization of the T96 model on Alfvén pulsation time of flight estimates. Figure 5 presents 
(a)

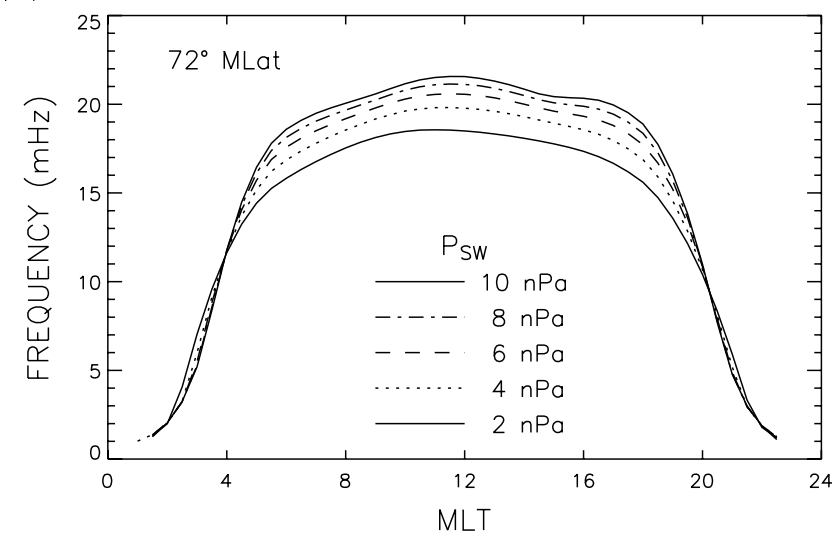

(b)

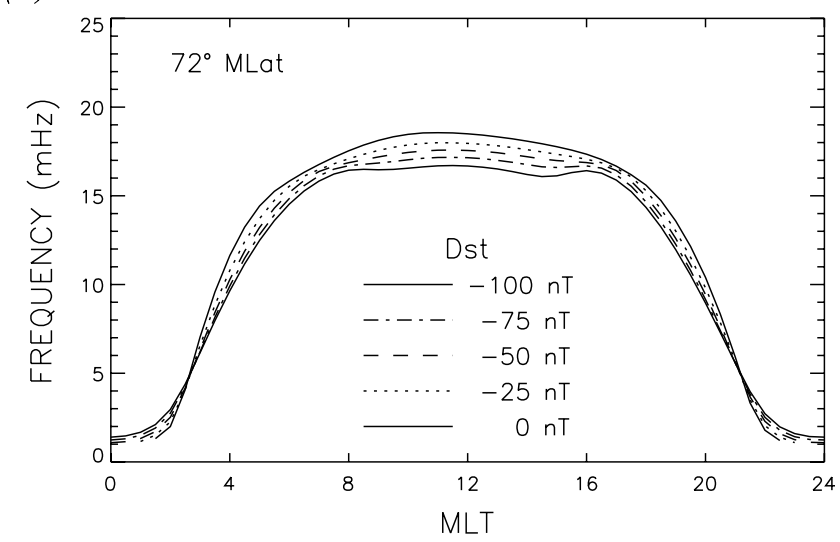

(c)

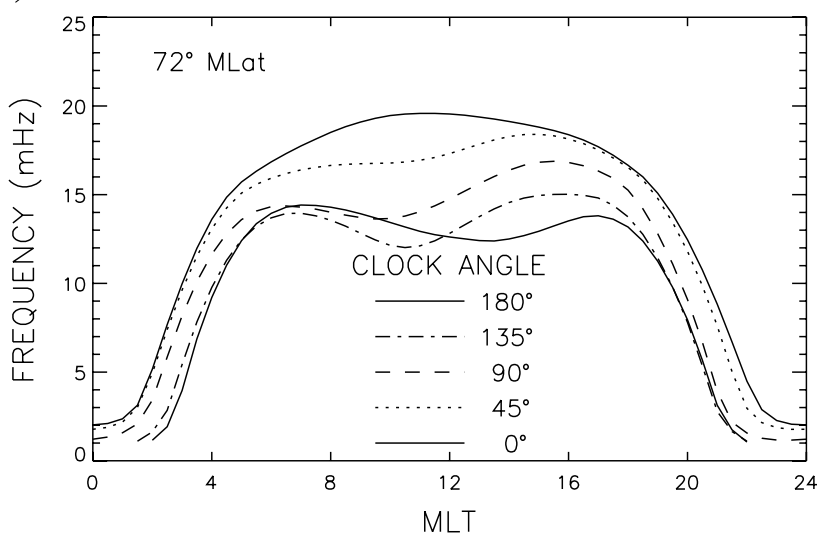

Figure 5. The results of varying the T96 input parameters when calculating the frequency of Alfvén pulsations standing on the $72^{\circ}$ Mlat magnetic field line under equinox conditions.

frequency-MLT profiles for pulsations on the $72^{\circ}$ Mlat field line under equinox conditions. In this figure a magnetospheric plasma density of 1 ion $\mathrm{cm}^{-3}$ at $4 R_{E}$ has been assumed. This enables the pulsation frequency to be scaled for any plasma number density by the relation $f=f_{0}(n)^{-\frac{1}{2}}$, where $f_{0}$ is the frequency read from the figure and $n$ the desired number density at $4 R_{E}$ in units of $\mathrm{cm}^{-3}$. The panels demonstrate the effects of adjusting the input parameters to the T96 model, namely $P_{S W}$ (Figure 5a), Dst (Figure 5b), and the IMF clock angle, defined as $\arctan \left(B_{Y} / B_{Z}\right)$ (Figure 5c).

[22] The effects of an increase in the solar wind dynamic pressure (Figure 5a) are broadly as expected. As $P_{S W}$ is increased from 2 to $10 \mathrm{nPa}$, the overall compression of the magnetosphere increases the pulsation frequency (reduces the pulsation period) at all local times and for all plasma density distributions. This effect is most significant between 0400 and 2000 MLT and is a consequence of (1) the generally reduced length of magnetospheric field lines and (2) the increased magnetic field strength in the compressed magnetospheric cavity resulting in faster pulsation propagation. We also note that in the midnight sector, field lines mapping to $72^{\circ}$ Mlat are "open" and therefore incapable of supporting standing waves.

[23] At most magnetic local times (0300-2100 MLT), the pulsation frequency tends to decrease as a function of (negatively) increasing Dst index (Figure 5b). This is as expected since the increasing magnitude of this input parameter is effectively increasing the strength of the ring current, resulting in a depressed geomagnetic field. The Alfvén speed in the inner magnetosphere is therefore also decreased, increasing the pulsation period and decreasing the frequency. In the night time sector, especially around midnight, the traced field lines extend far in the tail and are therefore less sensitive to changes in the Dst parameter.

[24] The rotation of the a fixed magnitude IMF $(|B|=$ $8 \mathrm{nT}$ ) about the Sun-Earth line, resulting in IMF clock angles between $0^{\circ}$ and $180^{\circ}$, dramatically alters the periodMLT profiles (Figure 5c). The approximate symmetry about the noon evident in previous figures was a consequence of the roughly symmetrical magnetic field configuration of the T96 magnetospheric field model with null IMF input parameterization (note that this is in contrast to the dawndusk symmetry built-in to the magnetic field model of Mead and Fairfield [1975] employed by Warner and Orr [1979]). However, as the IMF clock angle is rotated from northward pointing to southward, significant asymmetries are introduced into the magnetospheric configuration. The newly introduced asymmetry is characterized by a local minimum in the oscillation period that migrates from the morning to the afternoon sector as the IMF evolves from a northward to a southward orientation (via duskward). This is most likely an effect of the reconfiguration of the dayside magnetosphere due to the IMF-controlled interconnection of geomagnetic and solar wind magnetic fields across the magnetopause boundary (a feature of the T96 model not found in other data-based representations).

[25] Figure 6 presents the variations in pulsation frequency as a function of magnetic latitude along two magnetic local time meridians (0600 MLT: Figures 6a-6c and 1200 MLT: Figures 6d-6f). At each MLT, the three panels indicate the effect of varying the T96 input parameters exactly as in Figure 5. The same plasma density of 1 ion $\mathrm{cm}^{-3}$ at $4 R_{E}$ has also been retained. As such Figure 6 presents the latitudinal profile equivalent of the MLT profiles presented in Figure 5.

[26] As expected, we note that the variations in $P_{S W}$ result in moderate $(\sim 20 \%)$ frequency variations across the range of latitudes presented. The same is true for variations in the IMF clock angle, although in this case, the frequency variations are clearly more significant 

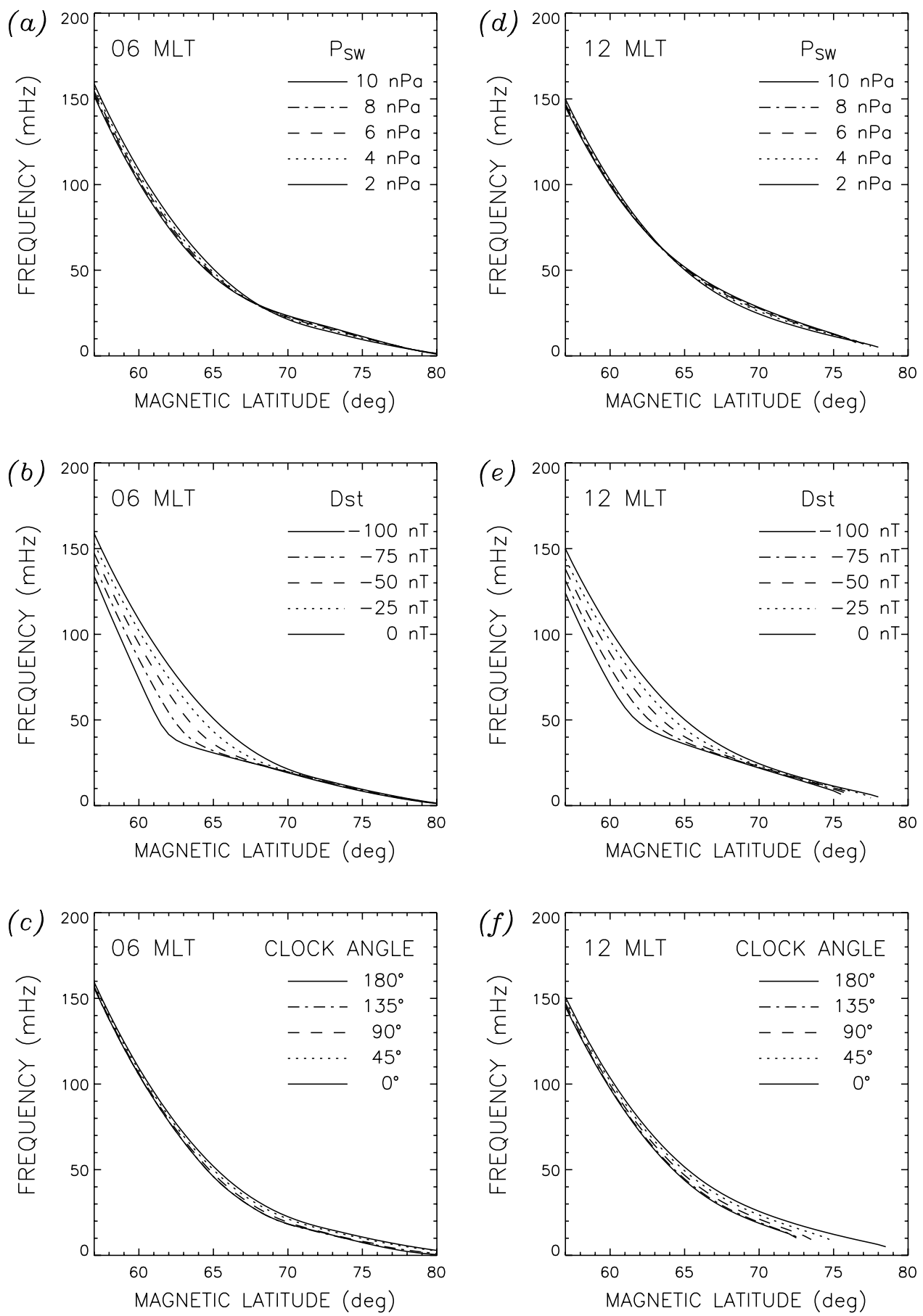

Figure 6. Variations in pulsation frequency as a function of magnetic latitude along the (a-c) 0600 MLT and $(\mathrm{d}-\mathrm{f}) 1200$ MLT meridians. Each panel indicates the effect of various input parameters upon the calculated pulsation frequency, as described in the text.

in the noon sector (Figure 6f) than in the dawn sector (Figure 6c) especially at $\sim 70^{\circ}-75^{\circ}$ Mlat. Some of the most significant effects on pulsation frequency are due to changes in the Dst parameter (Figures 6b and 6e). At midlatitudes $\left(57^{\circ}-65^{\circ}\right)$, adjustments to the Dst input parameter result in large variations in the estimated pulsation frequency (approaching a factor of 2 in some cases). Once again we note that this is a result of the increased magnetic field strength within the inner portion of the T96 magnetosphere due to the enhanced ring current.

\section{Discussion and Conclusions}

[27] The results presented above lead us to several clear conclusions. First, they validate the earlier results of Warner and $\operatorname{Orr}$ [1979]. We have, by utilizing an alternative magnetospheric magnetic field model, been able to broadly 
(a)

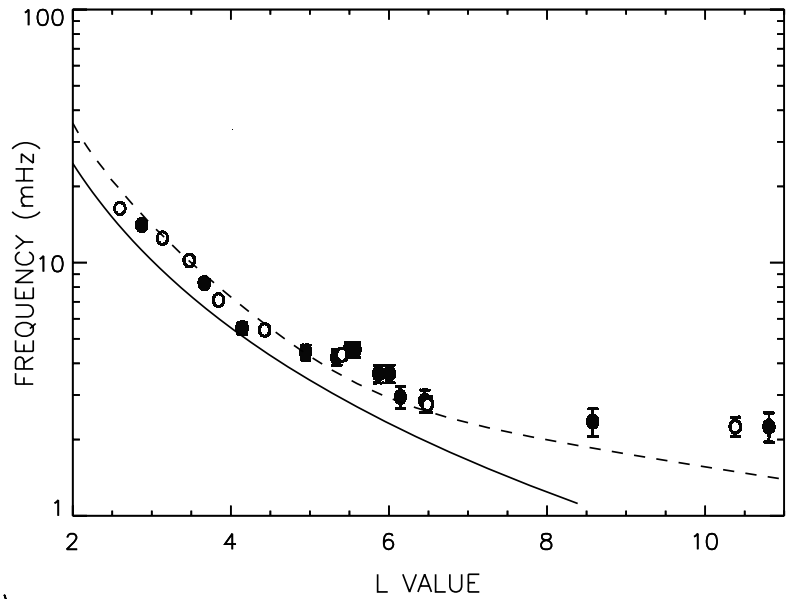

(b)

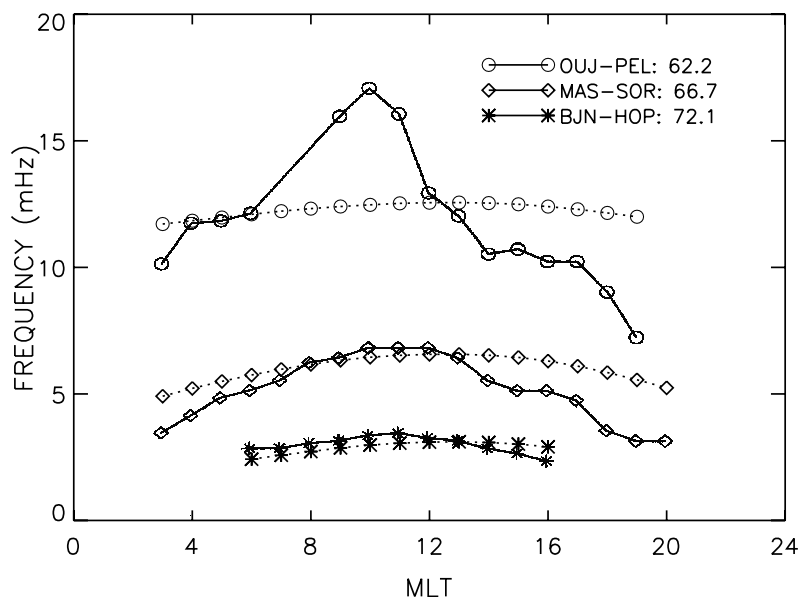

Figure 7. (a) Quiet-time field line resonance frequencies as a function of $L$ value for the interval $0700-0800 \mathrm{UT}$ on 1 January 1998, as presented by Menk et al. [2004]. These frequencies were determined via the twin-station crossphase (filled circles) and single-station power ratio analysis (unfilled circles) of ground-magnetometer data. (b) The diurnal variation of the Alfvén pulsation continuum (solid lines) derived via cross-phase analysis for three station pairs of the IMAGE ground magnetometer array, as presented by Mathie et al. [1999]. The symbols indicate the station pair used according to the key. Eigenfrequencies derived via the T96 time-of-flight technique are overlaid at the appropriate latitude (dotted lines).

reproduce the latitude-period and period-MLT profiles presented in that study. Second, while our finding are consistent with those of Warner and Orr [1979], we have extended our results to higher magnetic latitudes. Third, we have confirmed that upstream interplanetary conditions exert a powerful influence upon the period of Alfvénic pulsations in the magnetosphere.

[28] The first two conclusion are a consequence of the more realistic description of the magnetospheric magnetic field provided by the T96 model. The accurate representation of the extended magnetotail has enabled us to perform time-of-flight calculations at high latitudes, even in the midnight sector, with relative confidence.
[29] Perhaps more important though are the effects of the IMF orientation upon pulsation period in the dayside magnetosphere. For example, note the changes to morning/afternoon sector pulsation frequencies indicated in Figure 5c. This is in stark contrast to the period-MLT variations with null IMF parameterization (e.g., Figure 3b).

[30] Of course, the true test of this analysis technique is the comparison with observed ULF field-line oscillations. Figure 7 presents such a comparison. Figure 7a shows quiettime magnetic field line resonance frequencies as a function of $L$ value for the interval $0700-0800$ UT on 1 January 1998, as presented by Menk et al. [2004]. These frequencies were determined via the twin-station cross-phase analysis (filled circles) and single-station power ratio analysis (unfilled circles) of data from the SAMNET and IMAGE ground-magnetometer networks located in the Scandinavian sector. Overlaid on these data are frequency- $L$ shell profiles determined from the time-of-flight technique using T96 (dashed line) and dipolar (solid line) magnetic field geometries. In this case a plasma number density of 300 ions $\mathrm{cm}^{-3}$ and a $r^{-4}$ density variation was assumed. We note an encouraging systematic agreement between the observed frequencies and those estimated via the time-of-flight analysis. At the highest $L$ values (latitudes), we note that the calculation underestimates the observed pulsation frequency by $25 \%$. Given an identical plasma distribution, the T96 time-of-flight estimate is clearly superior the dipole field equivalent in the region poleward of $L=6$.

[31] Figure $7 \mathrm{~b}$ presents the diurnal variation of the Alfvén pulsation continuum (solid lines) derived via cross-phase analysis for three station pairs of the IMAGE ground magnetometer array, as presented by Mathie et al. [1999]. The symbols indicate the station pair used according to the key. Eigenfrequencies derived via the T96 time-of-flight technique are overlaid at the appropriate latitude (dotted lines). Once again, we note a generally good agreement between the estimated and observed frequencies. Perhaps surprisingly, the agreement between the two improves at increasing latitudes (where one might expect to be less confident in the modeled magnetic field geometry). This suggests that the discrepancy between predicted and observed pulsation frequency at the OUJ-PEL pair between 0600 and 1200 MLT is due to the inadequacy of the selected plasma model at midlatitudes in this MLT sector. It is difficult to account for the large changes in the observed frequency purely in terms of the variability of the magnetic field. It therefore seems likely that in this MLT/Mlat region, the magnetospheric plasma density has been significantly overestimated.

[32] This paper has demonstrated that the time-of-flight technique, combined with the T96 magnetic field model provides a useful method by which the eigenfrequency of geomagnetic field lines may be estimated. Although simplistic, this approach allows the investigation of highly stretched, asymmetric magnetospheric field lines for which no general analytical eigenmode calculations are available. The highly configurable nature of the technique described above, especially in terms of the dependency on universal time and upstream interplanetary conditions, permit the creation of bespoke time-dependent ULF pulsation period estimates for any chosen spacecraft or ground station, parameterized by realistic upstream interplanetary condi- 
tions. Comparison such as these will form the basis of future studies. In addition, a significant evolution of the analysis presented will arise from the implementation of a more realistic plasma density model, most likely derived from a recent magnetospheric satellite mission (such as Cluster or Double Star). We anticipate this to be a direction of future investigation also.

[33] Acknowledgments. During the course of this study, JAW was supported by PPARC rolling grant PPA/G/O/2003/0013.

34] Arthur Richmond thanks Karl-Heinz Glassmeier, John C. Samson, and two other reviewers for their assistance in evaluating this paper.

\section{References}

Ables, S. T., B. J. Fraser, C. L. Waters, D. A. Neudegg, and R. J. Morris (1988), Monitoring cusp/cleft topology using Pc5 ULF waves, Geophys. Res. Lett., 25, 1507-1510.

Allan, W., S. P. White, and E. M. Poulter (1986), Impulse-excited hydromagnetic cavity resonances in the magnetosphere, Planet. Space Sci., 34, $371-385$.

Anderson, B., M. J. Engebretson, and L. J. Zanetti (1989), Distortion effects in spacecraft observations of MHD toroidal standing waves: Theory and observations, J. Geophys. Res., 94, 13,425-13,445.

Anderson, B., M. J. Engebretson, S. P. Rounds, L. J. Zanetti, and T. A Potemra (1990), A statistical study of Pc-3 -5 pulsations observed by the AMPTE CCE magnetic-fields experiment, J. Geophys. Res., 95, 10,49510,523 .

Chappell, C. R. (1972), Recent satellite measurements of the morphology and dynamics of the plasmasphere, Rev. Geophys., 10, 951-979.

Chappell, C. R., K. K. Harris, and G. W. Sharp (1971), The dayside of the plasmasphere, J. Geophys. Res., 76, 7632-7647.

Clauer, C. R., A. J. Ridley, R. J. Sitar, H. J. Singer, A. S. Rodger, E. FriisChristensen, and V. O. Papitashvili (1997), Field line resonant pulsations associated with a strong dayside ionospheric shear convection flow reversal, J. Geophys. Res., 102, 4585-4596.

Cummings, W. D., R. J. O’Sullivan, and P. J. Coleman (1969), Standing Alfvén waves in the magnetosphere, J. Geophys. Res., 74, 778.

Dent, Z. C., I. R. Mann, F. W. Menk, J. Goldstein, C. R. Wilford, M. A. Clilverd, and L. G. Ozeke (2003), A coordinated ground-based and image satellite study of quiet-time plasmaspheric density profiles, Geophys. Res. Lett., 30(12), 1600, doi:10.1029/2003GL016946.

Dungey, J. W. (1954), Electrodynamics of the outer atmospheres, Rep. 69, Ions. Res. Lab., Penn. State Univ., University Park, Penn.

Glassmeier, K. H., and M. Stellmacher (2000), Concerning the local time asymmetry of Pc5 wave power at the ground and field line resonance widths, J. Geophys. Res., 105, 18,847-18,855.

Harrold, B. G., and J. C. Samson (1992), Standing ULF modes of the magnetosphere: A theory, Geophys. Res. Lett., 19, 1811-1814.

Kivelson, M. G., J. Etcheto, and J. G. Trotignon (1984), Global compressional oscillations of the terrestrial magnetosphere: The evidence and a model, J. Geophys. Res., 89, 9851-9856.

Lee, D. H., and R. L. Lysak (1990), Effects of azimuthal asymmetry on ULF waves in the dipole magnetosphere, Geophys. Res. Lett., 17, 53-56.

Loto'aniu, T. M., C. L. Waters, B. J. Fraser, and J. C. Samson (1999), Plasma mass density in the plasmatrough: Comparison using ULF waves and crres, Geophys. Res. Lett., 26, 3277-3280.

Mann, I. R., A. N. Wright, K. J. Mills, and V. M. Nakariakov (1999), Excitation of magnetospheric waveguide modes by magnetosheath flows, J. Geophys. Res., 104, 333-353.

Mathie, R. A., F. W. Menk, I. R. Mann, and D. Orr (1999), Discrete field line resonances and the Alfvén continuum in the outer magnetosphere, Geophys. Res. Lett., 26, 659-662.
Mead, G. D., and D. H. Fairfield (1975), A quantitative magnetospheric model derived from spacecraft magnetometer data, J. Geophys. Res., 100, $659-662$.

Menk, F. W., I. R. Mann, A. J. Smith, C. L. Waters, M. A. Clilverd, and D. K. Milling (2004), Monitoring the plasmapause using geomagnetic field line resonances, J. Geophys. Res., 109, A04216, doi:10.1029/ 2003JA010097.

Poulter, E. M., W. Allan, J. G. Keys, and E. Nielsen (1984), Plasmatrough ion mass densities determined from ULF pulsation eigenperiods, Planet. Space Sci., 32, 1069-1078.

Poulter, E. M., W. Allan, and G. J. Bailey (1988), ULF pulsation eigenperiods within the plasmosphere, Planet. Space Sci., 36, 185-196.

Rankin, R., F. Fenrich, and V. T. Tikhonchuk (2000), Shear Alfvén waves on stretched magnetic field lines near midnight in Earth's magnetosphere, Geophys. Res. Lett., 27, 3265-3268.

Rickard, G. J., and A. N. Wright (1994), Alfvén resonance excitation and fast wave propagation in magnetospheric waveguides, J. Geophys. Res., $99,13,455-13,464$

Samson, J. C., B. G. Harrold, J. M. Ruohoniemi, and A. D. M. Walker (1992), Field line resonance associated with MHD waveguides in the magnetosphere, Geophys. Res. Lett., 19, 441-444.

Singer, H. J., D. J. Southwood, R. J. Walker, and M. G. Kivelson (1981), Alfvén wave resonances in a realistic magnetospheric magnetic field geometry, J. Geophys. Res., 86, 4589-4596.

Sinha, A. K., and R. Rajaram (1997), An analytic approach to toroidal eigenmode, J. Geophys. Res., 102, 17,649-17,657.

Southwood, D. J. (1974), Some features of field line resonances in the magnetosphere, Planet. Space Sci., 22, 483-491.

Tsyganenko, N. A. (1995), Modeling the Earth's magnetospheric magnetic field confined within a realistic magnetopause, J. Geophys. Res., 100, $5599-5612$

Tsyganenko, N. A. (1996), Effects of the solar wind conditions on the global magnetospheric configuration as deduced from data-based field models, in Third International Conference on Substorms (ICS-3), ESA SP-389, pp. 181-185, Eur. Space Agency, Noordwijk, Netherlands.

Villain, J. P. (1982), Characteristics of Pc-5 micropulsations as determined with the stare experiment, J. Geophys. Res., 87, 129-137.

Warner, M. R., and D. Orr (1979), Time of flight calculations for highlatitude geomagnetic pulsations, Planet. Space Sci., 27, 679-689.

Waters, C. L., J. C. Samson, and E. F. Donovan (1995), The temporal variation of the frequency of high-latitude field line resonances, J. Geophys. Res., 100, 7987-7996.

Waters, C. L., J. C. Samson, and E. F. Donovan (1996), Variation of plasmatrough density derived from magnetospheric field line resonances, J. Geophys. Res., 101, 73724,746.

Waters, C. L., K. Takahashi, D.-H. Lee, and B. J. Anderson (2002), Detection of ultralow-frequency cavity modes using spacecraft data, J. Geophys. Res., 107(A10), 1284, doi:10.1029/2001JA000224.

Wright, A. N. (1992), Coupling of fast and Alfvén modes in realistic magnetospheric geometries, J. Geophys. Res., 97, 6429-6438.

Yeoman, T. K., and M. Lester (1990), Characteristics of mhd waves associated with storm sudden commencements observed by sabre and ground magnetometers, Planet. Space Sci., 38, 603-616.

Yumoto, K., and T. Saito (1983), Local time asymmetry in the characteristics of Pc5 magnetic pulsations, Planet. Space Sci., 31, 459-471.

C. L. Waters, School of Mathematical and Physical Sciences, University of Newcastle, Callaghan, NSW 2308, Australia.

J. A. Wild, Department of Communication Systems, InfoLab 21, Lancaster University, Lancaster, LA1 4WA, UK. (j.wild@lancaster.ac.uk)

T. K. Yeoman, Department of Physics and Astronomy, University of Leicester, Leicester LE1 7RH, UK. 